\title{
THE
}

3-15-1991

\section{Two-Dimensional Electronic Structure of the GaAs(110)-Bi System}

\author{
A. B. McLean \\ R. Ludeke \\ M. Prietsch \\ David R. Heskett \\ University of Rhode Island, dheskett@uri.edu \\ D. Tang
}

See next page for additional authors

Follow this and additional works at: https://digitalcommons.uri.edu/phys_facpubs

Terms of Use

All rights reserved under copyright.

\section{Citation/Publisher Attribution}

McLean, A. B., Ludeke, R., Prietsch, M., Heskett, D., Tang, D., \& Maeda Wong, T. (1991). Two-dimensional electronic structure of the GaAs(110)-Bi system. Physical Review B, 43(9), 7243-7253. doi: 10.1103/ PhysRevB.43.7243

Available at: http://dx.doi.org/10.1103/PhysRevB.43.7243

This Article is brought to you for free and open access by the Physics at DigitalCommons@URI. It has been accepted for inclusion in Physics Faculty Publications by an authorized administrator of DigitalCommons@URI. For more information, please contact digitalcommons-group@uri.edu. 


\section{Authors}

A. B. McLean, R. Ludeke, M. Prietsch, David R. Heskett, D. Tang, and T. Maeda Wong

This article is available at DigitalCommons@URI: https://digitalcommons.uri.edu/phys_facpubs/248 


\title{
Two-dimensional electronic structure of the $\operatorname{GaAs}(110)-B i$ system
}

\author{
A. B. McLean, ${ }^{*}$ R. Ludeke, and M. Prietsch ${ }^{\dagger}$ \\ IBM Thomas J. Watson Research Center, P.O. Box 218, Yorktown Heights, New York 10598 \\ D. Heskett and D. Tang \\ Department of Physics, University of Rhode Island, Kingston, Rhode Island 02881 \\ T. Maeda Wong \\ Department of Materials Science and Engineering, University of Pennsylvania, Philadelphia, Pennsylvania 19104
}

(Received 19 July 1990)

\begin{abstract}
The occupied electronic structure of the $\mathrm{GaAs}(110)-\mathrm{Bi}(1 \times 1)$ monolayer system has been studied using angle-resolved photoelectron spectroscopy with a synchrotron-radiation source. The overlayer system possesses at least three detectable surface states $\left(S^{\prime}, S^{\prime \prime}\right.$ and $\left.S^{\prime \prime \prime}\right)$ with two-dimensional character. Both the state with the lowest $\left(S^{\prime}\right)$ and the state with the highest $\left(S^{\prime \prime \prime}\right)$ binding energy are clearly visible over a large portion of the $(1 \times 1)$ surface Brillouin zone. The intermediate state $\left(S^{\prime \prime}\right)$ was observed along $\bar{\Gamma} \bar{X}^{\prime}$ and also in the neighborhood of $\bar{X}$. The intensity of all three states exhibits a predominantly $p_{z}$-like dependence on the polarization of the synchrotron light. However, $S^{\prime \prime \prime}$ possesses a greater component of $p_{x y}$-like character than either $S^{\prime}$ or $S^{\prime \prime}$. At the zone center, $S^{\prime}$ is situated $0.5 \mathrm{eV}$ above the valence-band maximum, and it disperses downwards by $\approx 1.0 \mathrm{eV}$ to $\bar{X}$, and by $\approx 0.8 \mathrm{eV}$ to $\bar{X}^{\prime}$. At $\bar{M}$ it has its binding-energy maximum, $1.3 \mathrm{eV}$ below the energetic position at $\bar{\Gamma}$. The two-dimensional electronic structure of this system is compared with that of the closely related $\mathrm{GaAs}(110)-\mathrm{Sb}(1 \times 1)$ monolayer system and with the results of first-principles calculations.
\end{abstract}

\section{INTRODUCTION}

At room temperature, most metal adsorbates react disruptively with the $\mathrm{GaAs}(110)$ surface, ${ }^{1}$ and consequently few form epitaxial monolayer systems. Therefore, although GaAs(110) is one of the most thoroughly characterized compound semiconductor surfaces (Ref. 2 and references therein), there are only a few adsorbates which produce overlayers that can be considered to be prototype or model systems. For example, it has recently been shown ${ }^{3}$ that $\mathrm{Sn}$ orders on $\mathrm{GaAs}(110)$. However, there appears to be no long-range, two-dimensional order, although the adlayer has a local $(3 \times 3)$ structure. The notable exceptions to the above rule appear to be elements from column $\mathrm{V}$ of the Periodic Table of which $\mathrm{Sb}$ is the best-known example. ${ }^{4-13} \mathrm{Bi}$ also orders on $\mathrm{GaAs}(110)$, and since it was demonstrated that $\mathrm{Bi}$ forms an ordered $(1 \times 1)$ overlayer on $\mathrm{GaAs}(110)$, several experimental studies have been performed ${ }^{14-22}$ on this system. These studies have provided a considerable amount of information about the occupied and unoccupied electronic structure of the semiconducting monolayer system and of the overlayer growth mode. However, the Bi-induced, twodimensional states have not been characterized as fully as the surface states of the $\mathrm{GaAs}(110)-\mathrm{Sb}(1 \times 1)$ monolayer system, ${ }^{7}$ although a preliminary investigation of the occupied surface-state band dispersion has been performed. ${ }^{14}$
Therefore, the GaAs(110)-Bi(1×1) monolayer system presents us with a useful opportunity to study the formation of an epitaxial, two-dimensional adlayer on $\mathrm{GaAs}(110)$. Since $\mathrm{Bi}$ has the same valence as $\mathrm{Sb}$, we expect the surface electronic structure to be similar. Moreover, since the nature of the adlayer-substrate bonding at the $\mathrm{GaAs}(110)-\mathrm{Sb}(1 \times 1)$ monolayer system has been the subject of considerable attention and discussion, it is possible that an investigation of the related $\mathrm{GaAs}(110)$ $\mathrm{Bi}(1 \times 1)$ monolayer system will shed light on the nature of the adatom-substrate bonding.

There are other reasons for studying this system which are related to the formation of the Schottky barrier at the metal-compound semiconductor interface. A previous investigation of the electrical properties of metalGaAs(110) Schottky contacts ${ }^{23}$ found that the Schottky barrier height of the Sb-GaAs(110) contacts was smaller than simple electronegativity considerations predicted. At that time, this behavior was anomalous and it was suggested $^{23}$ that a study of the related GaAs(110)-Bi system may help to establish whether or not this behavior was common to all semimetallic overlayers. It was also unclear whether or not popular theories of Schottky barrier formation should apply to semimetals as well as metals. Although we do not address the origin of the Schottky barrier at practical metal-semiconductor interfaces in this paper, we have discussed this subject elsewhere. ${ }^{14,21}$ 


\section{EXPERIMENTAL DETAILS}

The photoemission experiments were performed at beamline U12B of the National Synchroton Light Source (NSLS), Brookhaven National Laboratory, Upton, New York. The radiation from the storage ring was dispersed by a toroidal grating monochromator, ${ }^{24}$ which allowed the selection of light in the energy range from 10 to 140 $\mathrm{eV}$.

The GaAs(110) surfaces were prepared in a 14-in.diam, magnetically shielded, analysis chamber that possessed facilities for low-energy electron diffraction (LEED) and Ne-ion sputtering. The base pressure of the system was $1 \times 10^{-10}$ Torr, and during Bi evaporation the system pressure typically rose to $\approx 4 \times 10^{-10}$ Torr. A custom-built sample manipulator allowed the sample to be rotated about two axes. The GaAs single crystal was attached to a $\mathrm{Ta}$ plate mounted on a liquid-nitrogen dewar, which allowed the sample to be cooled to approximately $180 \mathrm{~K}$ and heated indirectly by passing a current through the Ta backing plate. The sample temperature was measured using a thermocouple that was attached to the sample holder near the sample. The optimum annealing conditions were determined using LEED. We also used photoemission to monitor the shape and intensity of the As-derived, dangling-bond surface state in the valence band, which was taken as a measure of the quality of the surface. The photoelectrons were collected with a hemispherical electrostatic energy analyzer, ${ }^{25}$ which had an angular acceptance of $\pm 2^{\circ}$. Our overall energy resolution was better than $200 \mathrm{meV}$. The analyzer was mounted on a goniometer which allowed the analyzer to be rotated about two orthogonal axes. This permitted full utilization of the polarized nature of the synchrotron radiation.

The azimuthal orientation of the $n$-type GaAs(110) wafer was determined to within $\pm 3^{\circ}$ using LEED. Throughout the experiment, the sample was aligned such that the [1 $\overline{1} 0]$ crystallographic direction was vertical, which positioned the Ga-As-Ga zig-zag chains vertically. Therefore the electric field vector of the incident light was parallel to the [001] direction (Fig. 1). The clean GaAs(110) surfaces were prepared using a sequence of sputtering-and-annealing cycles with Ne. Typically, the samples were sputtered using a defocussed beam of $1-\mathrm{kV}$ $\mathrm{Ne}$ ions for $30 \mathrm{~min}$ and subsequently annealed for 5-10 min at approximately $450^{\circ} \mathrm{C}$. Although the cleaved GaAs(110) surface is the surface of choice for most band-bending studies, if care is taken, ${ }^{26}$ reasonably highquality GaAs(110) surfaces can be produced by sputterand-annealing techniques. As a further check, we were able to compare our results with our earlier angleresolved photoemission studies of $\mathrm{Bi}$ overlayers on the cleaved GaAs(110) surface. ${ }^{14}$ Although the experiments were performed on another beam line, the monochromator that we used had comparable performance. Where direct comparison was possible between the clean, sputter-and-annealed surface and the clean, cleaved surface, we found that there were no significant differences in the relative intensity or position of features in the valence-band photoemission spectra. Furthermore, we were able to consistently prepare surfaces that had very similar valence-band spectra.

The overlayers were formed by evaporating $\mathrm{Bi}$ from a boron-nitride effusion cell onto a freshly prepared GaAs(110) surface, which was maintained at room temperature during deposition. The Bi overlayer thickness was calculated from timed exposures to the Bi evaporant beam, which was independently calibrated using a crystal-thickness monitor. We estimate the accuracy of
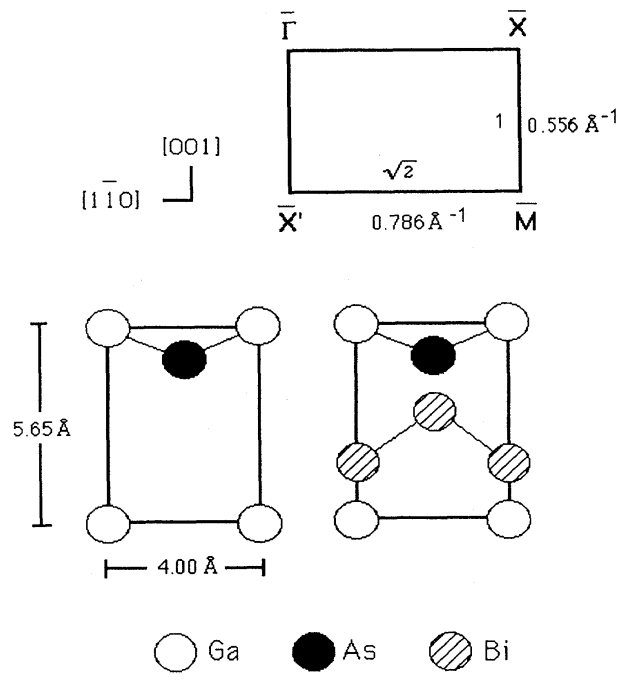

( a )

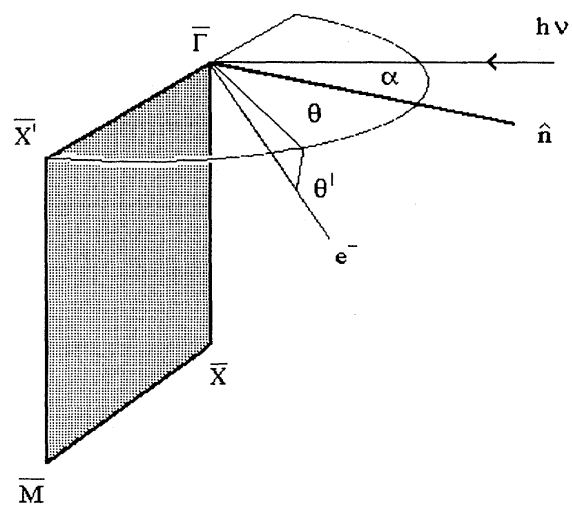

(b)

FIG. 1. (a) The surface Brillouin zone of $\mathrm{GaAs}(110)$ is presented in the upper figure. In the lower figure (left) the realspace unit cell of the GaAs(110) surface is shown and also (right) a possible configuration of the $\mathrm{Bi}$ atoms at $1 \mathrm{ML}$ (two atoms per unit cell). The covalent radii of $\mathrm{Ga}, \mathrm{As}$, and $\mathrm{Bi}$ are $1.26,1.20$, and $1.46 \AA$, respectively. For comparison, the covalent radius of $\mathrm{Sb}$ is $1.40 \AA$. (b) The experimental geometry: $\alpha$ is the angle of incidence, $\hat{\mathbf{n}}$ is the sample normal, and $\left(\theta, \theta^{\prime}\right)$ are the emission angles. 
the overlayer thickness to be better than $25 \%$, with a repeat accuracy of approximately $10 \%$. One monolayer (ML) is defined to be the coverage at which the atomic surface density of the adlayer equals the atomic surface density of the $\mathrm{GaAs}(110)$ surface $\left(8.854 \times 10^{14} \mathrm{~cm}^{-2}\right)$. We have assumed that the room-temperature sticking coefficient of $\mathrm{Bi}$ on the sample is identical to that of the thickness monitor.

As an aside, we also mention that core-level photoemission studies of the GaAs(110)-Bi system ${ }^{15,16}$ have shown that $\mathrm{Bi}$ desorbs from $\mathrm{GaAs}(110)$ at $\approx 350^{\circ} \mathrm{C}$. Consequently, we found that it was very easy to sputter or flash the Bi overlayers off the surface after we had completed our measurement cycle. We also found that the Bi overlayers were relatively inert and that they would remain clean for many hours at pressures of $2 \times 10^{-10}$ Torr. Consequently, Bi may serve as a useful capping material in applications where it would be disadvantageous to heat the $\mathrm{GaAs}(110)$ substrate above $350^{\circ} \mathrm{C}$.

\section{EXPERIMENTAL RESULTS}

\section{A. Bi-induced valence-band states}

In a previous paper, ${ }^{14}$ we presented the results of a preliminary angle-resolved photoemission investigation of the GaAs(110)-Bi system and reported the discovery of two Bi-induced states near the valence-band maximum of $\mathrm{GaAs}$, at Bi coverages of $1 \mathrm{ML}$. We also presented some estimates of the dispersion of these states along $\bar{\Gamma} \bar{X}$. Our major findings were that the uppermost Bi-induced state was located $\approx 0.3 \mathrm{eV}$ above the GaAs valence-band maximum at $\bar{\Gamma}$ and dispersed downwards by $0.5 \mathrm{eV}$ from $\bar{\Gamma}$ to $\bar{X}$. We also found that the uppermost state appears above the projected band structure of GaAs at all points in the surface zone along $\bar{\Gamma} \bar{X}$. In this study we have reexamined the initial-state dispersion of both states and extended our study to include a much larger portion of the surface Brillouin zone and a wider range of photon energies. We have also examined the development of these states as a function of Bi coverage. This has provided a much more complete picture of the $\mathrm{Bi}$-induced states.

In Fig. 1(a), the real-space and the reciprocal-space unit meshes ${ }^{27}$ are drawn for reference, and in Fig. 1(b) the experimental geometry is defined. In Fig. 2 we present two valence-band photoemission spectra taken in the neighborhood of the $\bar{X}$ point of the GaAs(110) surface Brillouin zone with $22-\mathrm{eV}$ light. The lower of the two curves was taken from the clean GaAs(110) surface, and the upper was taken from the ordered $\mathrm{GaAs}(110)$ $\mathrm{Bi}(1 \times 1)$ monolayer system. Before and after Bi deposition the Fermi level was situated near midgap $\pm 0.2 \mathrm{eV}$. The figure allows us to make a direct connection with our earlier work, ${ }^{14}$ which was performed predominantly with 22-eV light. It also illustrates the experimental difficulties that are often encountered with a conventional toroidal grating monochromator. The lower of the two spectra is dominated by three features. The feature that emerges at $1.1 \mathrm{eV}$ below the valence-band maximum $\left(A_{5}\right)$ is the As-derived surface state of the relaxed

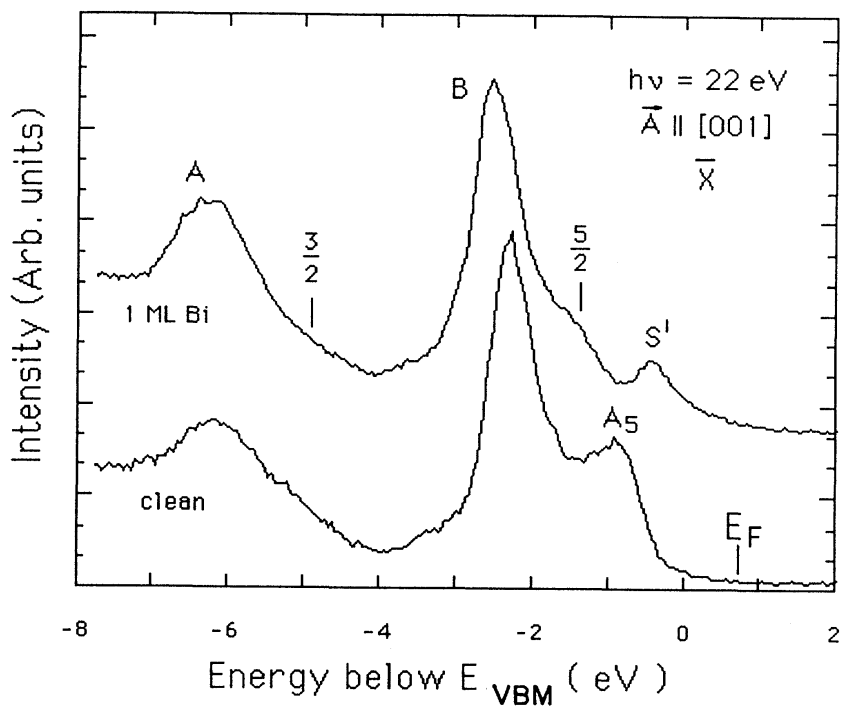

FIG. 2. Photoemission spectra from the clean GaAs(110) surface (lower curve) and from the same surface covered with 1 ML of Bi (upper curve). In both cases the uppermost states are probed at the $\bar{X}$ point of the surface Brillouin zone. The upper curve clearly shows the Bi-induced surface state $\left(S^{\prime}\right)$ which is split off from the bulk GaAs bands. In the upper curve, the arrows indicate the estimated positions of second-order features originating from the excitation of electrons from shallow $\mathrm{Bi} 5 d$ core levels with 44-eV light.

GaAs(110) surface. ${ }^{27}$ Using band-structure calculations ${ }^{28}$ we can use the position of $A_{5}$ at $\bar{X}$ to determine the energetic position of the valence-band maximum at $\bar{\Gamma}$, and we have assumed throughout this paper that $E_{\mathrm{VBM}}$ (at $\bar{\Gamma})-E\left(A_{5}\right.$ at $\left.\bar{X}\right)=1.1 \mathrm{eV}{ }^{28}$ This determination of the valence-band maximum should be accurate to within $\pm 0.1 \mathrm{eV}$ (see the discussion in Ref. 28). We have followed the convention of Ref. 29 and labeled the feature with the highest binding energy $A$.

In agreement with the results of our previous study ${ }^{14}$ Bi deposition causes a new Bi-induced state $\left(S^{\prime}\right)$ to appear at lower binding energy and at $\bar{X}$ it is clearly resolved. The spectrum from the monolayer system also contains two features which arise from the photoexcitation of electrons out of the $\mathrm{Bi} 5 d$ core level with secondorder, 44-eV light (labeled $\frac{3}{2}$ and $\frac{5}{2}$ in Fig. 2). The origin of the extra emission was verified by examining the photon-energy dependence of their binding energy. Although the feature originating from the $\mathrm{Bi} 5 d_{3 / 2}$ core level is very weak, at Bi coverages of $1 \mathrm{ML}$ the component originating from the $\mathrm{Bi} 5 d_{5 / 2}$ core level is quite strong, and it obscures a potentially interesting region of the valence band. However, little is to be gained by reducing the photon energy, as the second-order feature arising from the Ga $3 d$ level soon becomes a problem. Instead, we chose to investigate the photon-energy window above $22 \mathrm{eV}$. In Fig. 3 we present some of the results. The uppermost state of the monolayer system is probed at the $\bar{X}$ point with 22-, 30-, 32-, and 35-eV light. To facilitate comparison, the spectra have been normalized to incident photon flux and they are presented on a common 


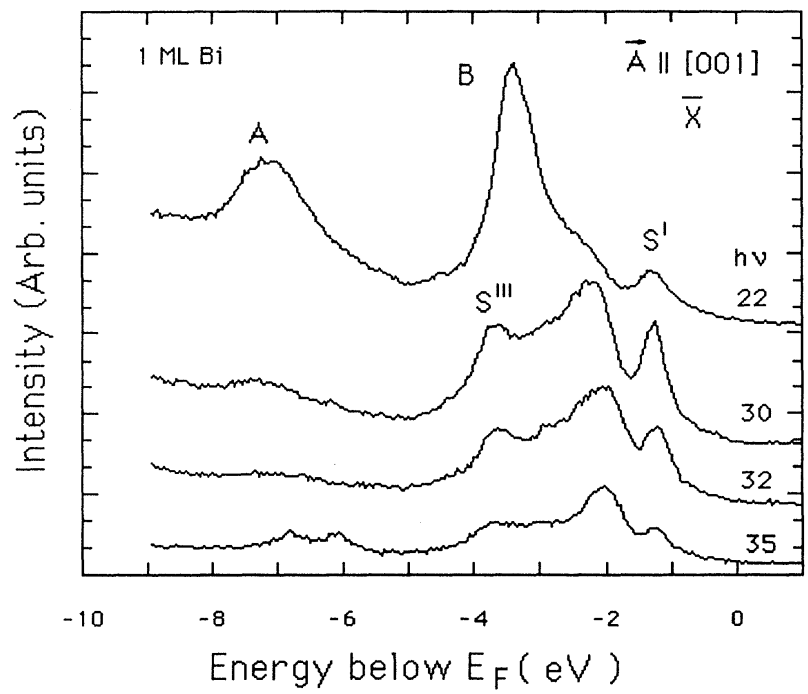

FIG. 3. The intensity of the Bi-induced valence-band features exhibit a strong dependence on the incident photon energy. This is illustrated in the photon energy range from 22 to $35 \mathrm{eV}$. In all four spectra, $S^{\prime}$ has been probed at the $\bar{X}$ point of the surface Brillouin zone. $S^{\prime}$ has an intensity maximum at approximately $30 \mathrm{eV}$, the energy that was chosen for most of the mapping. The spectra have been normalized to the incident photon flux. The two weak peaks which are visible in the lower spectrum, 6 and $7 \mathrm{eV}$ below the Fermi level, are the As $3 d$ core level in second order.

binding-energy scale. Notice that although the intensity of $S^{\prime}$ varies with photon energy, the binding energy remains constant, suggesting that $S^{\prime}$ is a two-dimensional electronic state. Also present in the 30-, 32-, and 35-eV spectra, are two additional, well-resolved states. The binding energy of the state that we have labeled $S^{\prime \prime \prime}$ remains constant as the photon energy is changed. The intermediate state $\left(S^{\prime \prime}\right)$ appears to shift to lower binding energy as the photon energy increases. This may be due to overlap with a bulk transition in this energy range.

Because of the strong similarity between the twodimensional electronic structure of the GaAs(110)-Bi and the GaAs(110)-Sb monolayer systems, the Bi-induced states have been labeled using the notation of Ref. 7 . This will simplify the discussion, Sec. IV. In our previous paper, we compared our experimental results with theoretical studies of the GaAs(110)-Sb system ${ }^{12,13}$ and referred to the uppermost state as $S_{6}\left(S^{\prime}\right)$ and the deeper of the two states as $S_{5}\left(S^{\prime \prime}\right)$.

The intensity of $S^{\prime}$ as a function of incident photon energy was examined and we found that the intensity of $S^{\prime}$ was maximum around photon energies of $30 \mathrm{eV}$. This also happens to be a photon-energy window where there are no serious second-order features in the valence band. $S^{\prime}$ is also well separated from other valence-band features and consequently the binding energy of this state can be determined relatively easily. In Fig. $4, S^{\prime}$ has once again been probed at the $\bar{X}$ point of the surface zone with $30-\mathrm{eV}$ light and the coverage dependence of the valence band has been examined in more detail. At $30 \mathrm{eV}$ the clean

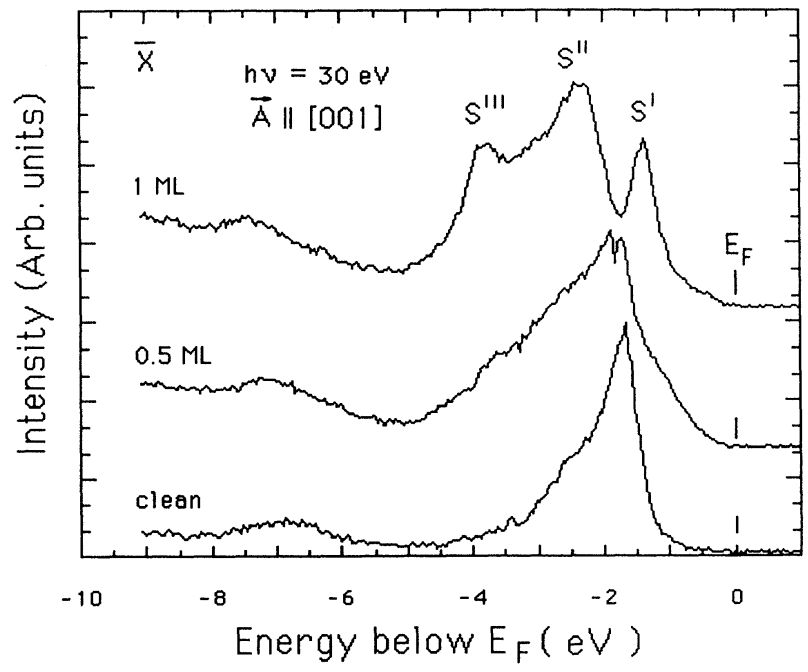

FIG. 4. With an incident photon energy of $30 \mathrm{eV}$ at $\bar{X}$, the valence-band spectra of $\mathrm{GaAs}(110)$ are dominated by a single feature. The deposition of $\mathrm{Bi}(0.5 \mathrm{ML})$ attenuates this feature in a systematic fashion and broadens the valence-band emission and subsequently produces (1 ML) three new features which are labeled $S^{\prime}, S^{\prime \prime}$, and $S^{\prime \prime \prime}$, respectively.

GaAs(110) valence-band photoemission spectrum is dominated by a single feature with a binding energy of $\approx 1.5$ eV. The shape of this spectrum at $\bar{X}$ is similar to the shape of the normal emission spectrum at the same photon energy, measured by Chiang et al. ${ }^{29}$ They assigned the strong feature in the neighborhood of the valenceband maximum to the photoexcitation of electrons from the bulk GaAs $\Sigma_{2}$ band. $^{29}$ When $\mathrm{Bi}$ is deposited $(0.5$ ML), this feature is attenuated, and the valence-band emission broadens to both lower and higher binding energy. At Bi coverages approaching $1 \mathrm{ML}$, the Bi-induced valence-band emission sharpens up into three wellresolved states ( $S^{\prime}, S^{\prime \prime}$, and $\left.S^{\prime \prime \prime}\right)$.

The two-dimensional character of $S^{\prime}$ and $S^{\prime \prime \prime}$ is verified in Fig. 5. At the $\bar{M}$ point of the surface Brillouin zone, the binding energy of $S^{\prime}$ and $S^{\prime \prime \prime}$ do not exhibit a noticeable dependence on the perpendicular component of the electron wave vector (i.e., photon energy). This strongly suggests, together with the results presented in Fig. 3, that both of these states have two-dimensional character. It should also be noted that the upper of the two spectra presented in Fig. 5, bears a resemblance to the $\bar{M}$ point, angle-resolved photoemission spectra taken from the InP(110)-Sb $(1 \times 1)$ monolayer system with unpolarized 21.2-eV light (Fig. 4 of Ref. 30).

\section{B. The $\bar{\Gamma} \bar{X}$ symmetry line}

So far, we have established that at least two surface states are present on the monolayer system that are not observed on the clean GaAs(110) surface. We have briefly discussed the photon-energy dependence of the uppermost state $\left(S^{\prime}\right)$ and the existence of second-order features produced by the relatively tight grouping of the 


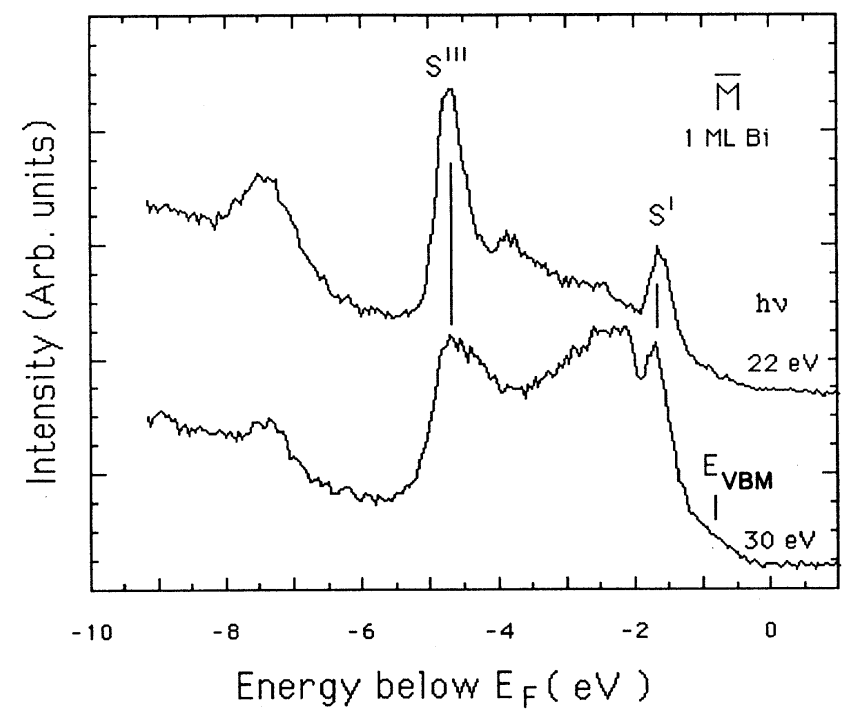

FIG. 5. $S^{\prime}$ is probed at the $\bar{M}$ point of the surface Brillouin zone with both $22-$ and $30-\mathrm{eV}$ light. The binding energies of $S^{\prime}$ and $S^{\prime \prime \prime}$ do not change appreciably in this photon-energy range. This behavior suggests that both of the electronic states possess two-dimensional character. The energy scale is referenced to the Fermi level and the position of the GaAs valence-band maximum is also shown.

Ga $3 d$, the $\mathrm{Bi} 5 d$, and the As $3 d$ core levels. It has been shown that the states denoted $S^{\prime}$ and $S^{\prime \prime \prime}$ are clearly resolved at two of the high-symmetry points of the surface zone $(\bar{X}$ and $\bar{M})$. It now remains to establish the band dispersion of these states over a larger portion of the surface zone and determine their polarization dependence.

The band dispersion of the surface states was determined in the conventional manner. ${ }^{31-33}$ The hemispherical analyzer was moved along the high-symmetry directions of the surface zone and the parallel component of the electron wave vector was calculated using the standard expression,

$$
\left|\mathbf{k}_{\|}\right|=\hbar^{-1}(2 m E)^{1 / 2} \sin \theta
$$

where $\left|\mathbf{k}_{\|}\right|$is the parallel component of the electronic wave vector, $m$ is the electronic mass, $E$ is the kinetic energy of the photoelectron, and $\theta$ is the takeoff angle, the angle subtended by the detector and the sample normal [see Fig. 1(b)].

As an example, we present in Fig. 6, a series of spectra which were obtained by mapping along the $\bar{\Gamma} \bar{X}$ line from the $\bar{X}$ point of the surface Brillouin zone to the $\bar{\Gamma}$ point of the second zone $\left(\bar{\Gamma}_{2}\right)$. The $\bar{\Gamma} \bar{X}$ direction is perpendicular to the GaAs(110) mirror plane and along the line of the Ga-As-Ga chains. $S^{\prime}$ is clearly resolved in this portion of the surface zone and the state disperses upwards from $\bar{X}$ to both $\bar{\Gamma}_{1}$ (not shown) and $\bar{\Gamma}_{2}$ (Fig. 6). Although $S^{\prime \prime \prime}$ is easily resolved at the high-symmetry points $\left(\bar{X}\right.$ and $\left.\bar{\Gamma}_{2}\right)$, we were not able to study its dispersion in any detail with 30-eV light in the region from $\bar{X}$ to $\bar{\Gamma}_{2}$. In the neighborhood of $\bar{\Gamma}_{2}$, the state we have labeled $S^{\prime \prime}$ is clearly visible $\approx 0.4 \mathrm{eV}$ below $S^{\prime}$. Notice that since $S^{\prime}$ has its binding energy minimum at $\bar{\Gamma}$, Fig. 6 clearly shows that the Fermi level coincides with the emission maximum. Analogous results were obtained earlier using the scanning tunneling microscope (STM) ${ }^{14}$ On $p$-type samples the Fermi level is pinned at the bottom of a semiconductorlike energy gap of magnitude $\approx 0.7 \mathrm{eV}$, which lies within the GaAs energy gap. On cleaved $n$-type GaAs(110) surfaces the Fermi level is located $0.2 \mathrm{eV}$ higher. It has been shown that there are acceptorlike states within the monolayer bandgap and these states appear at the edges of the Bi terraces. ${ }^{14}$ On the sputter-and-annealed surface we would expect the distribution of electronic states at midgap to be different. Therefore, the fact that we find the Fermi level position $0.1-0.2 \mathrm{eV}$ higher within the gap on the sputter-and-annealed surface does not cause us concern. We also observed some variation of the order of $\pm 0.1 \mathrm{eV}$ in the Fermi level position from one clean surface to another.

The dispersion of $S^{\prime}$ has been plotted against $\left|\mathbf{k}_{\|}\right|$in Fig. 7. Also included is the dispersion of the dominant bulk transition labeled $B$, which is observed with $22-\mathrm{eV}$ light, calculated using the procedure outlined above. Because this is a bulk feature, it is not required to have the symmetry of the surface zone as observed in the figure. The dispersion of $S^{\prime}$ along the $\bar{\Gamma} \bar{X}$ line was obtained using 22-eV light, in the neighborhood of $\bar{X}$, and using 30$\mathrm{eV}$ light from $\bar{X}$ to $\bar{\Gamma}_{2}$. Also shown is the projection of the GaAs bulk bands from a recent calculation using a first-principles Green's function approach. ${ }^{28}$ In agreement with the results of our earlier study, ${ }^{14} S^{\prime}$ appears above the projected bulk bands at all points along $\bar{\Gamma} \bar{X}$ and it has its binding-energy maximum at the zone boundary.

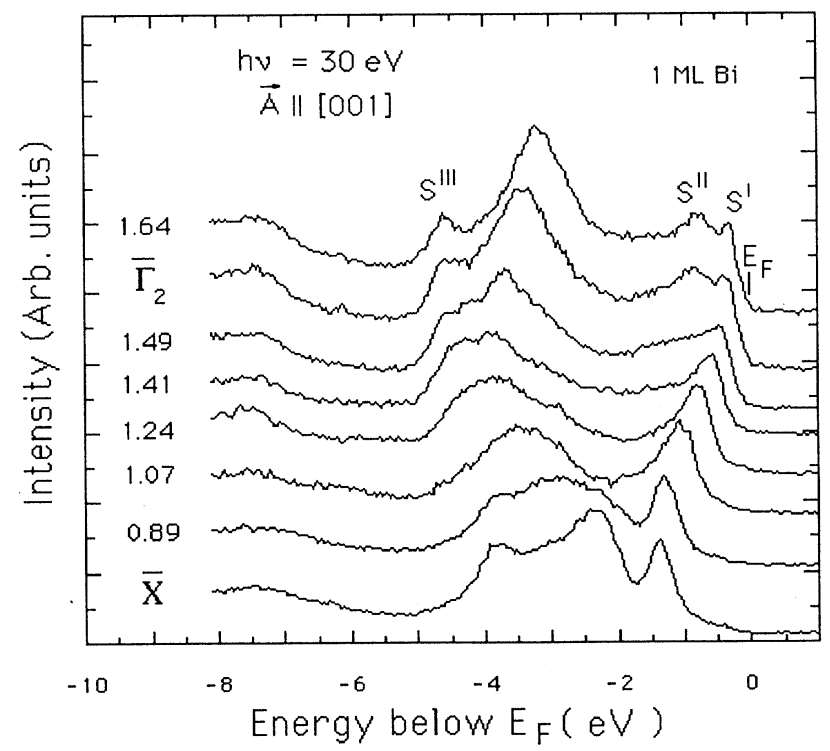

FIG. 6. $S^{\prime}$ is mapped from $\dot{\bar{X}}$ to $\bar{\Gamma}_{2}$ with $30-\mathrm{eV}$ light. At $\bar{\Gamma}_{2}$ $S^{\prime \prime}$ is also visible, $\approx 0.4 \mathrm{eV}$ below $S^{\prime}$. $S^{\prime \prime \prime}$ is visible at both of the high-symmetry points $\left(\bar{X}\right.$ and $\left.\bar{\Gamma}_{2}\right)$. The parallel component of the electron wave vector is indicated on each spectrum in units of $\AA$. $\bar{X}$ and $\bar{\Gamma}_{2}$ are probed at $\approx 0.79$ and $1.57 \AA-1$, respectively. 


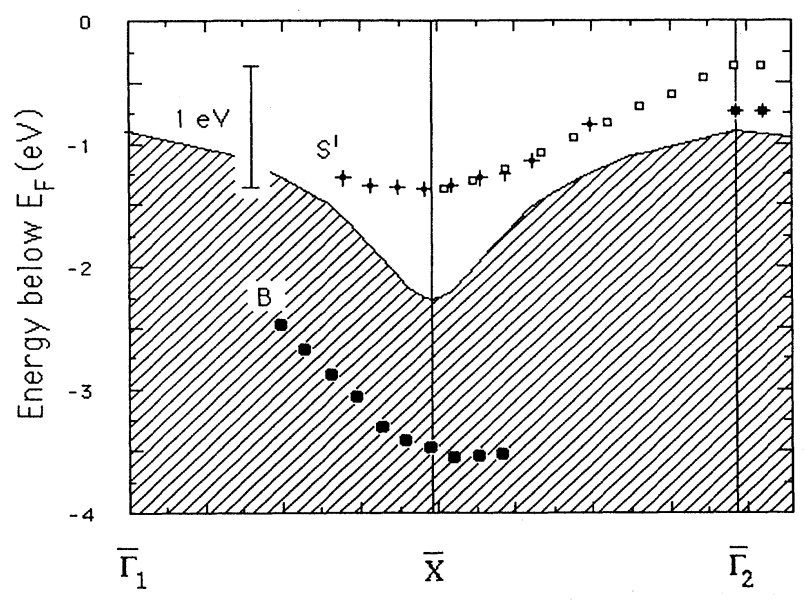

FIG. 7. The dispersion of $S^{\prime}$ along the $\bar{\Gamma} \bar{X}$ line. Also included is a feature known to originate from a bulk transition (Ref. 14 and references therein). It is the feature labeled $B$ in Fig. 2, and it does not possess the symmetry of the surface Brillouin zone. Also shown is the projected bulk bands which have recently been calculated (Ref. 28) using a first-principles Green's function approach.

\section{The $\bar{\Gamma} \bar{X}^{\prime}$ symmetry line}

In Fig. 8, we present five valence-band photoemission spectra which were collected along $\bar{X}^{\prime} \bar{\Gamma}_{2}$ with $30-\mathrm{eV}$ light (upper panel) and one valence-band spectrum which was collected at $\bar{X}^{\prime}$ (lower panel). The spectra clearly illustrate the three states, which we have labeled $S^{\prime}, S^{\prime \prime}$, and $S^{\prime \prime \prime}$. Within our experimental resolution of $0.2 \mathrm{eV}, S^{\prime}$ and $S^{\prime \prime}$ appear to be degenerate at $\bar{X}^{\prime}$. This is interesting because existing theoretical studies of the GaAs(110)$\mathrm{Sb}(1 \times 1)$ system $^{12,13}$ predict that the two states should be nondegenerate at this point in the surface zone. Moreover, as $\bar{\Gamma}_{2}$ is approached from $\bar{X}^{\prime}, S^{\prime}$ and $S^{\prime \prime}$ merge, and it becomes increasingly difficult to determine the binding energies of the states without resorting to line-shape analysis.

\section{D. $\bar{X} \bar{M}, \bar{M} \bar{X}^{\prime}$ and the surface Brillouin zone}

In Secs. III B and III C, the dispersion of the surface states has been investigated along the two main symmetry lines of the surface Brillouin zone. We also mapped these states along the edge of the surface zone, from $\bar{X}$ to $\bar{X}^{\prime}$ via $\bar{M}$. The analyzer was moved such that $\left|\mathbf{k}_{\|}\right|$lay on the zone boundary and the azimuthal orientation of the sample was the same as we had used previously ( $\mathbf{A} \|[001]$; see Fig. 1(b)).

The dispersion of states $S^{\prime}, S^{\prime \prime}$, and $S^{\prime \prime \prime}$ around the edge of the surface Brillouin zone, and also along $\bar{\Gamma} \bar{X}$ and $\bar{\Gamma} \bar{X}^{\prime}$, is summarized in Fig. 9 . Although we were able to confirm that the dispersion of $S^{\prime}$ and $S^{\prime \prime}$ is the same, within experimental error, when measured with both 22and 30-eV light at a number of points in the surface zone, we were unable to resolve $S^{\prime \prime \prime}$ with $22-\mathrm{eV}$ light. We attribute this to overlap with bulk transitions. Furthermore, all of the Bi-induced states are clearly visible over a large portion of the surface zone outside the bulk continuum,
TABLE I. Surface-state binding energies at high-symmetry points. Energies are in units of electron volts $( \pm 0.1 \mathrm{eV})$ and expressed relative to the GaAs valence-band maximum. The energetic positions, relative to the Fermi level, can be obtained using $E_{F}-E_{\mathrm{VBM}}=0.89 \mathrm{eV}$.

\begin{tabular}{lccr}
\hline \hline & $S^{\prime}$ & $S^{\prime \prime}$ & $S^{\prime \prime \prime}$ \\
\hline $\bar{\Gamma}$ & -0.54 & -0.11 & +3.72 \\
$\bar{X}$ & +0.44 & +1.44 & +2.69 \\
$\bar{M}$ & +0.72 & +1.23 & +3.71 \\
$\bar{X}^{\prime}$ & +0.29 & +0.29 & +2.32 \\
\hline \hline
\end{tabular}

split off from the GaAs bulk bands. The surface-state binding energies are summarized in Table $\mathrm{I}$.

\section{E. Surface-state polarization dependence probed at $\bar{X}$}

By using the polarized nature of synchrotron radiation, we were able to determine the polarization dependence of the Bi-induced surface features at $\bar{X}$. By moving the hemispherical analyzer below the incident synchrotron light beam, a wide range of polarization geometries could be accessed by rotating the sample and the analyzer to-

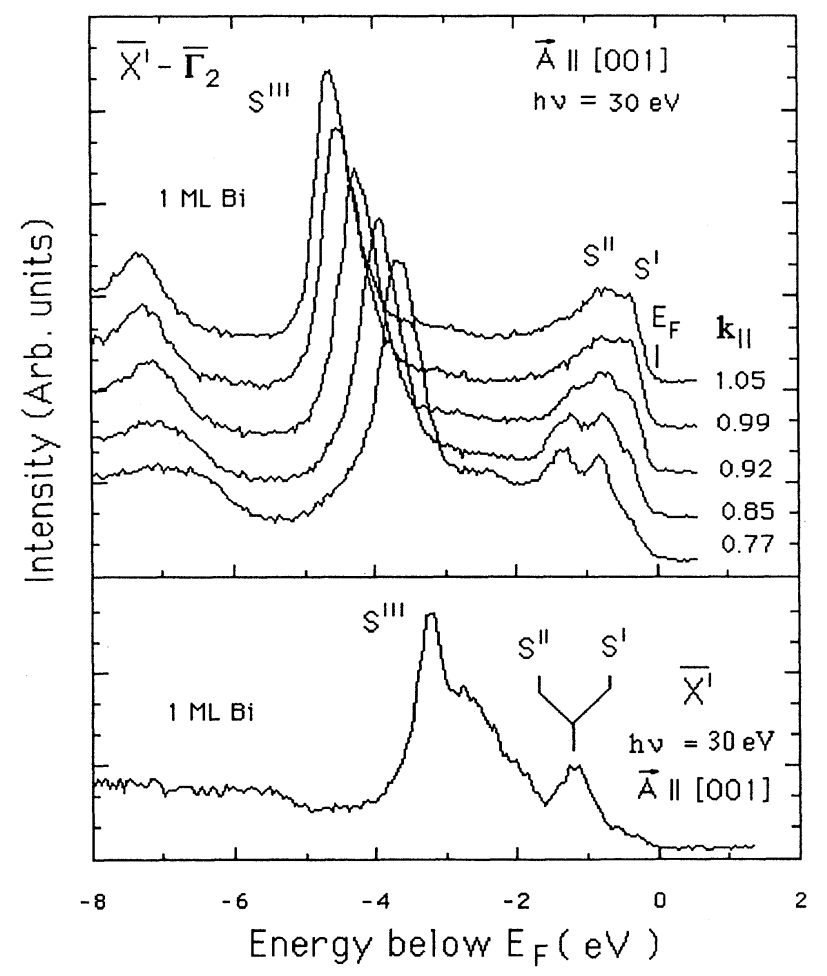

FIG. 8. The upper five valence-band photoemission spectra were taken between $\bar{X}^{\prime}$ and $\bar{\Gamma}_{2}$ along $\bar{X}^{\prime} \bar{\Gamma}_{2}$. The value of the parallel momentum is indicated on each spectrum in units of 1 $\AA$. Along this direction the three observable surface states are clearly visible. $\bar{X}^{\prime}$ and $\bar{\Gamma}_{2}$ are probed at $\approx 0.56$ and $1.11 \AA^{-1}$, respectively. The lower spectrum was taken with $30-\mathrm{eV}$ light at $\bar{X}^{\prime}$ and it is included to illustrate that $S^{\prime}$ and $S^{\prime \prime}$ appear to be degenerate at $\bar{X}^{\prime}$. 


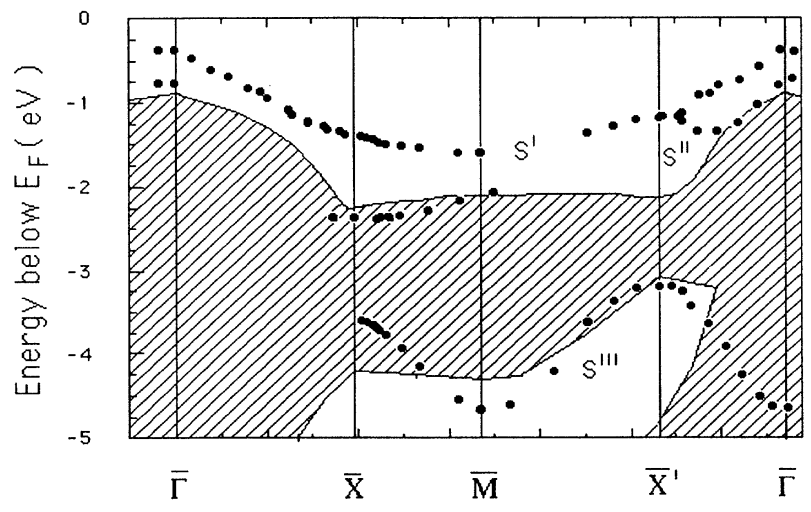

FIG. 9. The dispersion of the three Bi-induced surface states $S^{\prime}, S^{\prime \prime}$, and $S^{\prime \prime \prime}$ are presented around the $(1 \times 1)$ surface Brillouin zone. Also shown is the projected bulk bands (Refs. 28).

gether. In Fig. 10, we present four valence-band photoemission spectra which have been normalized to the incident photon flux. In the lowest curve $(\alpha=0)$, the synchrotron light was incident normal to the surface. Therefore, the component of the electric field vector perpendicular to the surface is zero. Under these conditions, $S^{\prime}$ is still visible. The same behavior was demonstrated by the uppermost surface state of the $\mathrm{GaAs}(110)-\mathrm{Sb}(1 \times 1)$ monolayer system (Fig. 4 of Ref. 7). With $\mathrm{Bi}$, although neither $S^{\prime \prime}$ nor $S^{\prime \prime \prime}$ are resolved with $\alpha=0$, it can still be argued that they exhibit some emission intensity at normal incidence. This suggests that although all of the states are predominantly $p_{z}$-like, none of the three states are purely $p_{z}$-like, even allowing for the fact that the light may not be completely polarized (Table II). The sample and the analyzer were then rotated by $20^{\circ}$, relative to the

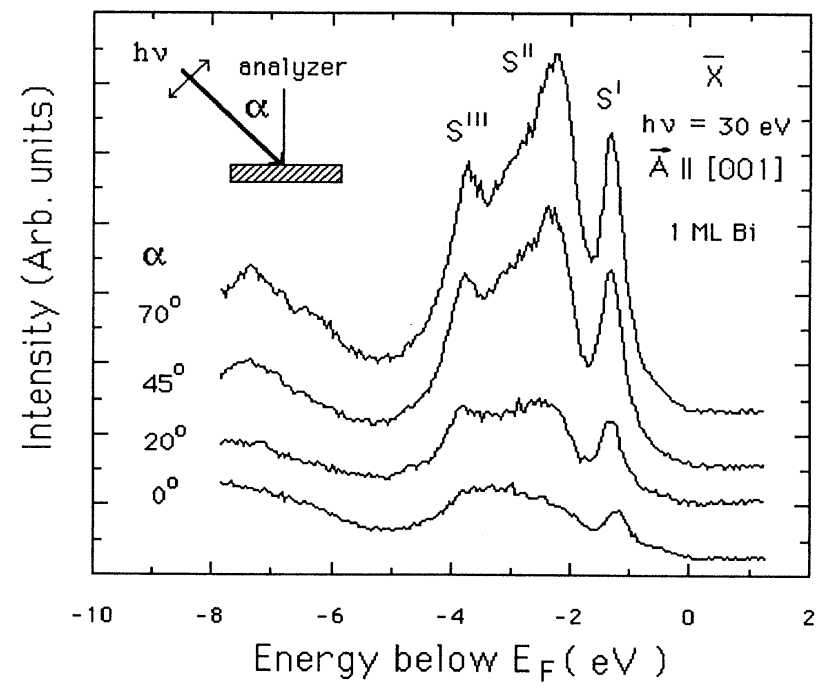

FIG. 10. The polarization dependence of the valence-band features is probed at the $\bar{X}$ point of the surface zone. $\alpha$ is the angle of incidence. Assuming $100 \%$ polarization of the light, $\alpha=0^{\circ}$ is completely $s$ polarized and $\alpha=70^{\circ}$ is mainly $p$ polarized.
TABLE II. Polarization dependence of surface states.

\begin{tabular}{lcc}
\hline \hline Surface state & $\begin{array}{c}\text { GaAs(110)-Sb }(1 \times 1) \\
\text { Character }\end{array}$ & Reference \\
\hline$S^{\prime}$ & $p_{z}$ & Ref. 6 \\
$S^{\prime \prime}$ & $p_{z}$ & Ref. 6 \\
$S^{\prime \prime \prime}$ & $p_{x y}$ & Ref. 6 \\
& GaAs(110)-Bi(1)1) & \\
Surface state & Character & Reference \\
\hline$S^{\prime}$ & $p_{z}$ & Present study \\
$S^{\prime \prime}$ & $p_{z}$ & Present study \\
$S^{\prime \prime \prime}$ & $p_{z}$ & Present study \\
\hline \hline
\end{tabular}

incident light beam and, once again, $S^{\prime}$ was probed at the $\bar{X}$ point. As the perpendicular component of the electric field vector increases, so does the intensity of all three surface states.

In Fig. 11, the intensity of $S^{\prime}$ has been plotted against the angle of incidence $(\alpha)$. Also shown is a curve of form $\left(\sin ^{2} \alpha+\beta \cos ^{2} \alpha\right)$, where $\beta=0.15$. A similar analysis was performed on the other two states as the value of $\beta$ can be used as a measure of the $p_{x y}$ contribution to the predominantly $p_{z}$-like states. The results are presented in Table III.

\section{DISCUSSION}

In the preceding section, we demonstrated that the $\operatorname{GaAs}(110)-\mathrm{Bi}(1 \times 1)$ monolayer system possesses at least three detectable Bi-induced states $\left(S^{\prime}, S^{\prime \prime}\right.$, and $\left.S^{\prime \prime \prime}\right)$, which are not present on the clean GaAs(110) surface, and are visible over a reasonably large portion of the sur-

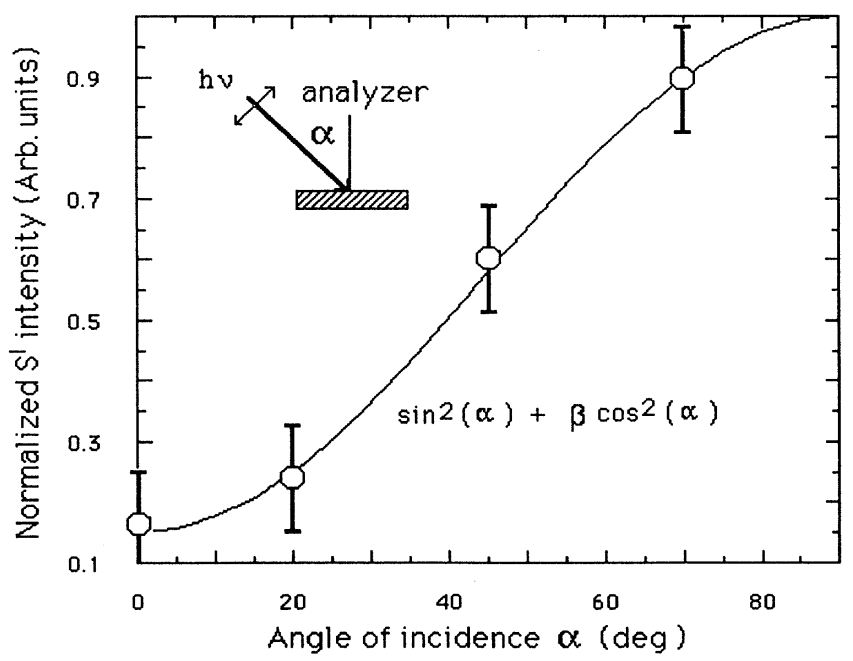

FIG. 11. The normalized intensity of the Bi-induced surface state $S^{\prime}$ is plotted against $\alpha$, the angle of incidence. The experimental geometry is included. The analyzer is placed below the sample normal at $\bar{X}$. A trial function of form $\sin ^{2}(\alpha)+\beta \cos ^{2}(\alpha)$, with $\beta=0.15$, adequately models the polarization dependence of the surface-state intensity. 
TABLE III. Ratio of $p_{x y}$ to $p_{z}$-like character $(\beta)$.

\begin{tabular}{ll}
\hline \hline$S^{\prime}$ & $0.15 \pm 0.05$ \\
$S^{\prime \prime}$ & $0.15 \pm 0.05$ \\
$S^{\prime \prime \prime}$ & $0.25 \pm 0.05$ \\
\hline \hline
\end{tabular}

face Brillouin zone. The intensity of all three states demonstrates a predominantly $p_{z}$-like dependence on the polarization of the synchrotron light. The dispersion of the surface states along the orthogonal $\bar{\Gamma} \bar{X}$ and $\bar{\Gamma} \bar{X}^{\prime}$ directions and the similarity with the GaAs(110)$\mathrm{Sb}(1 \times 1)$ system $^{6}$ suggests that the $\mathrm{Bi}$ overlayer is truly two-dimensional. The band dispersion of these states suggest that there is significant wave-function overlap along both symmetry directions. This may arise from direct interaction with the neighboring $\mathrm{Bi}$ atoms or indirect interaction mediated by the substrate.

As mentioned above, we presented in Ref. 14 the results of a preliminary angle-resolved photoemission investigation of the GaAs(110)-Bi(1×1) monolayer system, in which we presented an estimate of the initial-state energy dispersion of two surface states $\left(S_{5}\right.$ and $\left.S_{6}\right)$ along $\bar{\Gamma} \bar{X}$. However, in this paper, we have reported finding only one dominant surface state $\left(S^{\prime}\right)$ along this symmetry line. This is because our present studies of the Bi-coverage dependence of these states have led us to believe that $S_{5}$ is actually a remnant of the As-derived dangling-bond surface state $\left(A_{5}\right)$ and not a new Bi-induced state. Also, in the current investigation, we have performed most of our surface-state mapping outside the first surface zone. We found that the Bi-induced states are more clearly separated from the bulk emission features in the second surface zone. Consequently, we found that the bandwidth of $S^{\prime}$ along the $\bar{\Gamma} \bar{X}$ symmetry line was larger than we had originally ascertained. ${ }^{14}$

Although we did not examine the low-energy electron diffraction patterns in any detail, the patterns that we did obtain from the monolayer system were consistent with our earlier LEED studies. ${ }^{14,22}$ The overlayer has the $(1 \times 1)$ symmetry of the GaAs(110) surface with additional superlattice spots, indicating that there is additional order parallel to the Ga-As-Ga chains. The additional spots are consistent with the notion that the overlayer contains an ordered array of missing $\mathrm{Bi}$ atoms every 24-25 $\AA$. This overlayer structure has in fact been observed with the scanning tunneling microscope (STM) by Feenstra. ${ }^{14,21}$ We argued previously ${ }^{14}$ that this adlayer structure arises as a result of lattice-mismatch-induced strain in the $\mathrm{Bi}$ terraces. The $\mathrm{Bi}$ atoms appear to nucleate randomly and grow along [110] until they reach the strain-limited critical length of $24-25 \AA$. This picture has been confirmed by some recent LEED studies. ${ }^{17,18}$ Although STM has provided a considerable amount of information about this surface structure, there is always the concern that the STM is probing only a small portion of the surface. In this case the LEED studies $^{17,18}$ have confirmed that the satellite diffraction spot is a sixthorder spot. This means that the $24-\AA$ periodicity, observed by Feenstra ${ }^{14,21}$ is a characteristic length of the adlayer over length scales of $\approx 500 \AA$. It has also been found ${ }^{17,18}$ that the satellite spots are more elongated than the integral order spots, as if there is some variation in the position of the end points of the chains. Once again this result is consistent with the STM images of the Bi terraces on $\operatorname{GaAs}(110) .{ }^{14,21}$ As far as our angle-resolved photoemission studies are concerned, the GaAs(110)$\mathrm{Bi}(1 \times 1)$ monolayer system is indistinguishable from a true $(1 \times 1)$ system. So far we have not been able to identify photoemission features that may arise from the formation of a $(6 \times 1)$ superlattice. The LEED studies have also confirmed the result that, in contrast to $\mathrm{Sb}$, the structural order of the $\mathrm{GaAs}(110)-\mathrm{Bi}(1 \times 1)$ system is not significantly improved by annealing. As has been pointed out before, ${ }^{22}$ this result is not surprising since bulk Bi has a substantially lower melting point $\left(271^{\circ} \mathrm{C}\right)$ than bulk $\mathrm{Sb}$ $\left(631^{\circ} \mathrm{C}\right)$ and the structural defects in the overlayer may anneal out at room temperature.

This angle-resolved photoemission study complements recent inverse photoemission studies of the same system. ${ }^{20,22}$ We found previously ${ }^{22}$ that at Bi coverages of 1 ML the cation-derived empty surface state $\left(C_{3}\right)$ is replaced by two unoccupied, Bi-induced states 0.9 and 1.9 eV above the valence-band maximum of GaAs at $\bar{\Gamma}$. From their coverage-dependent intensities, we assigned these two states to the outer (Bi-Bi) and the inner [GaAs(110)-Bi] interfacial layers, respectively. We also found that both states exhibited resonant behavior. There was an enhancement of the photon intensity when the emitted photon energy equals the surface plasmon energy. This kind of plasmon resonance was first observed $^{34}$ during an inverse photoemission investigation of the $\mathrm{GaAs}(110)-\mathrm{Sb}(1 \times 1)$ monolayer system. Although we had observed layer-dependent states with inverse photoemission and expected to find similar behavior on the occupied side of the Fermi level, we found no evidence for layer-dependent surface states above $1 \mathrm{ML}$ in the present photoemission study.

The angular dependence of the inverse photoemission spectra has recently been studied by $\mathrm{Hu}$ et al. ${ }^{20}$ They found that the feature that dominates the 1-ML inverse photoemission spectra, situated $\approx 1.9 \mathrm{eV}$ above $\bar{\Gamma}$ in normal emission, ${ }^{22}$ disperses upwards by $0.2 \mathrm{eV}$ from $\bar{\Gamma}$ to $\bar{X}$, but exhibits no detectable dispersion along the $\bar{\Gamma} \bar{X}^{\prime}$ symmetry line. The unoccupied electronic structure of the $\mathrm{GaAs}(110)-\mathrm{Sb}(1 \times 1)$ monolayer system had previously been found to behave in the same fashion. ${ }^{35}$ In the case of $\mathrm{GaAs}(110)-\mathrm{Bi}(1 \times 1)$, the authors relate this behavior ${ }^{20}$ to the formation of quasi-one-dimensional zig-zag $\mathrm{Bi}$ chains along [ $1 \overline{1} 0]$ and suggest that the lack of dispersion along $\bar{\Gamma} \bar{X}^{\prime}$ was a consequence of weak Bi-Bi interaction perpendicular to the $\mathrm{Bi}$ chains. In contrast, our data conclusively demonstrate that the GaAs(110)-Bi monolayer system is two dimensional.

Although no attempt has yet been made to calculate the surface electronic structure of the GaAs(110)$\mathrm{Bi}(1 \times 1)$ monolayer system, the close similarity between our results and those of Ref. 7 suggest that the existing theoretical studies of the GaAs(110)-Sb(1×1) system should provide a convenient framework to interpret our own experimental results. However, we proceed with caution. It would be substantially more satisfactory to be 
able to compare our results directly with a theoretical calculation of the surface electronic structure of the $\operatorname{GaAs}(110)-\mathrm{Bi}(1 \times 1)$ monolayer system.

The two most recent theoretical studies of the GaAs(110)-Sb(1 $1 \times 1)$ monolayer system ${ }^{12,13}$ have both predicted the existence of six $\left(S_{1}-S_{6}\right)$ occupied Sbderived electronic states and two unoccupied $\mathrm{Sb}$-derived electronic states $\left(S_{7}, S_{8}\right)$. Although the predicted interfacial bonding configuration is different in both cases, the surface-state binding energies at the high-symmetry points of the surface zone are in reasonable agreement with each other. The first calculation ${ }^{12}$ was performed with the pseudopotential method using surface-atomic geometries that had been extracted from a dynamical analysis of the LEED intensities. ${ }^{5}$ The second calculation was performed using an empirical tight-binding approach. The surface-atomic geometries were obtained using energy minimization and they were very close to the geometry favored by the analysis of the LEED data. ${ }^{5}$ Both studies agree that the states with the highest binding energy $\left(S_{1}\right.$ and $\left.S_{2}\right)$ are $s$-like states which are localized on the $\mathrm{Sb}$ adatoms. However, the two studies ${ }^{12,13}$ propose different assignments for the remaining states. In Ref. 12 it is suggested that $S_{3}$ and $S_{4}$ are backbonds produced by the covalent bonding of the $\mathrm{Sb}$ atoms with the $\mathrm{Ga}$ and As $s p^{3}$ hybrids. The unoccupied states, $S_{7}$ and $S_{8}$, are the antibonding counterparts. It is also suggested that $S_{5}$ and $S_{6}$ are localized in the $\mathrm{Sb}$ adlayer and that they are basically $p$-like, dangling-bond, $\mathrm{Sb}$ orbitals pointing out of the surface plane. Therefore their photoemission intensity should have a $p_{z}$-like dependence on the polarization of the synchrotron light. In contrast, it is proposed in Ref. 13 that $S_{3}$ and $S_{4}$ are $p^{2}$ bonding states which lie within the Sb-Sb chain and that $S_{7}$ and $S_{8}$ are the antibonding counterparts. The authors also suggest that a $\pi$ bond between the $p_{z}$ state normal to the $\mathrm{Sb}$ chains and the $s p^{3}$ hybrids of the GaAs substrate produces states $S_{5}$ and $S_{6}$. We would, therefore, expect in this case as well $S_{5}$ and $S_{6}$ to demonstrate a $p_{z}$-like polarization dependence in photoemission. This bonding configuration resolved a puzzle (e.g., Ref. 13) which arose from the analysis of the LEED data, ${ }^{5}$ namely that $p^{2}$ bonding within the $\mathrm{Sb}$ chain occurred simultaneously with $s p^{3}$-like bond angles to the substrate.

As we mentioned earlier, both calculations predict similar binding energies for the Sb-induced surface states at the high-symmetry points of the surface zone. Therefore, we would not expect that we would be able to distinguish between these two bonding configurations using our experimental energy dispersions. We can, however, make some general observations about the surface-state dispersion and examine the polarization dependence of the $\mathrm{Bi}$ induced states and compare them with the expected polarization dependence of the $\mathrm{Sb}$-induced states.

First, ignoring a rigid shift in the experimentally determined band dispersion to lower binding energy by $\approx 0.5$ $\mathrm{eV}$, we note that the states we have designated $S^{\prime}$ and $S^{\prime \prime}$ most closely resemble $S_{6}$ and $S_{5} \cdot{ }^{12,13}$ Furthermore, the band dispersion of $S^{\prime}$ strongly resembles the band dispersion of the $S^{\prime}$ state of the GaAs(110)-Sb $(1 \times 1)$ monolayer system. ${ }^{7}$ The major difference is the energetic position relative to the bulk-band edges. However, $S^{\prime \prime}$ seems to behave differently in the two systems. For example, in Ref. 7 it was found for $\operatorname{GaAs}(110)-\mathrm{Sb}$ that $S^{\prime}$ is well separated from $S^{\prime \prime}$ at the $\bar{X}^{\prime}$ point of the surface zone. In contrast, for $\operatorname{GaAs}(110)-\mathrm{Bi}$, we found that within our experimental resolution $S^{\prime}$ and $S^{\prime \prime}$ appear to be degenerate at $\bar{X}^{\prime}$. Furthermore, we could only resolve one state along $\bar{X}^{\prime} \bar{M}$. We emphasize this point because it appears to be a major difference between the two epitaxial monolayer systems.

Although there are some significant differences between the initial-state band dispersion of the three surface states that we have examined and similar states found at the $\mathrm{GaAs}(110)-\mathrm{Sb}(1 \times 1)$ monolayer system, the most striking difference is the observed polarization dependence of the surface states. As mentioned above, we have determined that all three of the surface states have predominantly $p_{z}$-like character, with possibly some $p_{x y}{ }^{-}$ like character. The fact that $S^{\prime}$ and $S^{\prime \prime}$ have $p_{z}$-like character is not surprising, since both theoretical investigations of the GaAs(110)-Sb $(1 \times 1)$ monolayer system ${ }^{12,13}$ predict that $S_{5}$ and $S_{6}$ should have $p_{z}$-like character. However, the theoretical studies predict that $S_{3}$ should either arise from a backbond ${ }^{12}$ or from an in-plane $\mathrm{Sb}-$ $\mathrm{Sb}$ bond. ${ }^{13}$ In the latter case we would expect the state to have $p_{x y}$-like character as observed by Martensson et al. ${ }^{7}$ In the former case we may expect $S_{3}$ to possess mixed character.

\section{CONCLUSIONS}

In the preceding sections we have presented the results of our angle-resolved photoemission study of the GaAs(110)-Bi(1×1) monolayer system. All evidence points to the fact that the $\mathrm{Bi}$ atoms do not react disruptively with the GaAs(110) surface but rather from ordered terraces at submonolayer coverages and an ordered $(1 \times 1)$ overlayer at coverages of $1 \mathrm{ML}$. However, both STM and LEED studies have detected the existence of a $(6 \times 1)$ superlattice superimposed upon the $(1 \times 1)$ structure. The superlattice is produced by missing rows of $\mathrm{Bi}$ atoms aligned along the [001] crystallographic direction. The missing rows have been imaged directly with the STM $^{14,21}$ and indirectly with LEED. ${ }^{17,18,22}$ The LEED patterns have sixfold, satellite spots which indicate that there is additional order along [110] with a periodicity of $24 \AA$. We have suggested elsewhere ${ }^{14}$ that the missing rows of $\mathrm{Bi}$ atoms relieve lattice-mismatch-induced strain in the $\mathrm{Bi}$ adlayer. Therefore, it would be of interest to study Bi overlayers on III-V semiconductor surfaces that are better lattice matched to $\mathrm{Bi}$, such as $\operatorname{InAs}(110)$ or $\mathrm{GaSb}(110)$.

The photoemission spectra demonstrate that there are at least three new Bi-induced surface states. Similar states with similar initial-state energy dispersion (ignoring a rigid shift of $\approx 0.5 \mathrm{eV}$ ) are observed at the GaAs(110)-Sb $(1 \times 1)$ system. Therefore due to the similar valence of the two atoms, the same physical factors may influence the formation of the two interfaces. However, we have found that even though the initial-state disper- 
sion of these three states is similar, the polarization dependence of one of the states is different. We have found that $S^{\prime \prime \prime}$ possesses predominantly $p_{z}$-like character whereas the $S^{\prime \prime \prime}$ state of the GaAs(110)-Sb(1×1) monolayer system has been found to have $p_{x y}$ character. ${ }^{7}$ The physical reason for this is not understood, and since it implies a structural difference in the two systems, it would be of considerable interest to study the polarization dependence of the Sb-induced surface states at the $\mathrm{GaAs}(110)-\mathrm{Sb}(1 \times 1)$ monolayer system in more detail. It is possible that the larger size of the $\mathrm{Bi}$ atom stabilizes a different bonding configuration. For example, we already know that the larger size of the $\mathrm{Bi}$ atom gives rise to missing rows of $\mathrm{Bi}$ atoms and it is reasonable to suppose that there may be structural differences between the two systems.

Finally, the results presented in this paper may open up the discussion about the adlayer-substrate bonding mechanism at the $\mathrm{GaAs}(110)-\mathrm{Sb}(1 \times 1)$ monolayer system and for reference we have presented in Table II, the experimentally determined surface-state character for both $\mathrm{GaAs}(110)-\mathrm{Sb}(1 \times 1)$ and $\mathrm{GaAs}(110)-\mathrm{Bi}(1 \times 1)$. In Table I, we have presented the binding energies of the $\mathrm{Bi}$ induced electronic states, relative to the valence-band maximum of GaAs. From these figures, the surface-state bandwidths along the major symmetry directions can easily be extracted. Furthermore, in Table III, we have listed the value of $\beta$ for all three states. $\beta$ represents the amount of $p_{x y}$-like character that the state possesses. The values suggest that all three surface states have predominantly $p_{z}$-like character. Although $S^{\prime}$ and $S^{\prime \prime}$ are most probably purely $p_{z}$-like, our results suggest that $S^{\prime \prime \prime}$ contains a larger component of $p_{x y}$ character, consistent with the notion that the orbital is not aligned along the surface normal.

\section{ACKNOWLEDGMENTS}

The experiments were performed at the Penn-Oak Ridge National Laboratory (ORNL) photoemission beamline (U12) at the National Synchrotron Light Source, Brookhaven National Laboratory. We would like to thank P. Bruhwiler, B. Itchkawicz, and M. Prikas for experimental assistance during our run at U12. It is also our pleasure to acknowledge discussions with F. J. Himpsel and R. M. Feenstra. One of us (D.H.) would also like to acknowledge partial support from the Department of Energy Division of University and Industry Programs, Office of Energy Research.
*Permanent address: Department of Physics, Queen's University, Kingston, Ontario, Canada K7L 3N6.

$\dagger$ Permanent address: Institut fur Atom-und Festkorperphysik, Freie Universitat Berlin, D-1000, Berlin 33, Germany.

${ }^{1}$ J. F. McGilp and A. B. McLean, J. Phys. C 21, 807 (1988).

${ }^{2}$ A. Kahn, Surf. Sci. Rep. 3, 193 (1983); C. B. Duke and A. Patton, Surf. Sci. 164, L797 (1985).

${ }^{3}$ C. K. Shih, Efthimios Kaxiras, R. M. Feenstra, and K. C. Pandey, Phys. Rev. B 40, 10044 (1989).

${ }^{4}$ P. Skeath, C. Y. Su, W. A. Harrison, I. Lindau, and W. E. Spicer, Phys. Rev. B 27, 6246 (1983).

${ }^{5}$ C. B. Duke, A. Paton, W. K. Ford, A. Kahn, and J. Carelli, Phys. Rev. B 26, 803 (1982).

${ }^{6} \mathrm{~J}$. R. Myron, J. Anderson, and G. J. Lapeyre, in Proceedings of the 17th International Conference on the Physics of Semiconductors, edited by J. D. Chadi and W. A. Harrison (SpringerVerlag, New York, 1985), p. 133.

${ }^{7}$ P. Mårtensson, G. V. Hansson, M. Låhdeniemi, K. O. Magnusson, S. Wiklund, and J. M. Nichols, Phys. Rev. B 33, 7399 (1986); P. Mårtensson, Ph.D. thesis, Linkoping University, Sweden, 1986 (unpublished).

${ }^{8}$ A. Tulke, M. Mattern-Klosson, and H. Luth, Solid State Commun. 59, 303 (1986).

${ }^{9}$ F. Schäffler, R. Ludeke, A. Taleb-Ibrahimi, G. Hughes, and D. Rieger, Phys. Rev. B 36, 1328 (1987); J. Vac. Sci. Technol. B 5, 1048 (1987).

${ }^{10}$ R. M. Feenstra and P. Mårtensson, Phys. Rev. Lett. 61, 447 (1988).

${ }^{11}$ P. Mårtensson and R. M. Feenstra, Phys. Rev. B 39, 7744 (1989).

${ }^{12}$ C. M. Bertoni, C. Calandra, F. Manghi, and E. Molinari, Phys. Rev. B 27, 1251 (1983); F. Manghi, C. Calandra, and E. Molinari, Surf. Sci. 184, 449 (1987).
${ }^{13}$ C. Mailhiot, C. B. Duke, and D. J. Chadi, Phys. Rev. Lett. 53, 2114 (1984); Phys. Rev. B 31, 2213 (1985).

${ }^{14}$ A. B. McLean, R. M. Feenstra, A. Taleb-Ibrahimi, and R. Ludeke, Phys. Rev. B 39, 12925 (1989).

${ }^{15}$ J. J. Joyce, J. Anderson, M. M. Nelson, C. Yu, and G. J. Lapeyre, J. Vac. Sci. Technol. A 7, 850 (1989).

16J. J. Joyce, J. Anderson, M. M. Nelson, and G. J. Lapeyre, Phys. Rev. B 40, 10412 (1989).

${ }^{17}$ T. Guo, R. E. Atkinson, and W. K. Ford, Rev. Sci. Instrum. 61, 968 (1989).

${ }^{18}$ T. Guo, R. E. Atkinson, and W. K. Ford, Phys. Rev. B 41, 5138 (1990).

${ }^{19}$ G. D. Waddill, C. M. Aldao, C. Capasso, P. J. Benning, Yongjun $\mathrm{Hu}$, T. J. Wagener, M. B. Jost, and J. H. Weaver, Phys. Rev. B 41, 5960 (1990).

${ }^{20}$ Yongjun $\mathrm{Hu}$, T. J. Wagener, M. B. Jost, and J. H. Weaver, Phys. Rev. B 40, 1146 (1989); 41, 5817 (1990).

${ }^{21}$ R. Ludeke, A. Taleb-Ibrahimi, R. M. Feenstra, and A. B. McLean, J. Vac. Sci. Technol. B 7, 936 (1989).

${ }^{22}$ A. B. McLean and F. J. Himpsel, Phys. Rev. B 40, 8425 (1989).

${ }^{23}$ A. B. McLean and R. H. Williams, J. Phys. C 21, 783 (1988); A. B. McLean, R. H. Williams, and J. F. McGilp, Solid State Commun. 65, 1415 (1988); J. Vac. Sci. Technol. B 6, 1252 (1988).

${ }^{24}$ B. P. Tonner, Nucl. Instrum. Methods 172, 133 (1980).

${ }^{25}$ C. L. Allyn, T. Gustafsson, and E. W. Plummer, Rev. Sci. Instrum. 49, 1197 (1978).

${ }^{26}$ P. Oelhafen, J. L. Freeouf, G. D. Pettit, and J. M. Woodall, J. Vac. Sci. Technol. B 1, 787 (1983).

${ }^{27}$ G. V. Hansson and R. I. G. Uhrberg, Surf. Sci. Rep. 9, 197 (1988), and references therein.

${ }^{28}$ X. Zhu, S. B. Zhang, S. G. Louie, and M. L. Cohen, Phys. 
Rev. Lett. 63, 2112 (1989). The position of the central gap was not discussed by the above authors. So in Fig. 9 we positioned it relative to the band edges using the calculations of $\mathbf{J}$. R. Chelikowsky and M. L. Cohen, Phys. Rev. B 20, 4150 (1970).

${ }^{29}$ T. C. Chiang, J. A. Knapp, D. E. Eastman, and M. Aono, Solid State Commun. 31, 917 (1979).

${ }^{30}$ C. Maani, A. McKinley, and R. H. Williams, J. Phys. C 18, 4975 (1985).
${ }^{31}$ F. J. Himpsel, Adv. Phys. 32, 1 (1983).

${ }^{32}$ E. W. Plummer and W. Eberhardt, Adv. Chem. Phys. 49, 533 (1982).

${ }^{33}$ N. V. Smith, in Angular Dependent Photoemission, Photoemission in Solids I, edited by M. Cardona and L. Ley (SpringerVerlag, Berlin, 1978).

${ }^{34}$ W. Drube, F. J. Himpsel, and P. J. Feibelman, Phys. Rev. Lett. 60, 2070 (1988).

${ }^{35}$ W. Drube and F. J. Himpsel, Phys. Rev. B 37, 8551 (1988). 